\title{
Analysis of the clinical and aesthetic results of facial dimple creation surgery
}

\author{
Jae Min Chung ${ }^{1}$, Joo Hyuk Park², Jeong Su Shim² \\ ${ }^{1}$ V Aesthetic Plastic Surgical Clinic, Daegu; ${ }^{2}$ Department of Plastic and Reconstructive Surgery, Daegu Catholic University School of Medicine, \\ Daegu, Korea
}

Background Dimples on the cheeks can make the smile look more cheerful and attractive. Therefore, some people who do not have dimples may choose to undergo dimple creation surgery. Although dimple surgery is quite common, those desiring this procedure often lack information about it. Therefore, we conducted the present study to share our surgical tips and clinical experiences regarding safe dimple creation surgery.

Methods This study included 2,048 patients who underwent dimple creation surgery at our plastic surgery clinic between April 2010 and June 2014. These patients were selected from those who displayed no scarring from injury or tumor removal in the central face during the presurgical evaluation. Medical records were used to identify the age and sex of each patient, the location of dimple creation, any postoperative complications, reoperation, and the reason for reoperation.

Results Of the 2,048 patients, 159 (7.7\%) underwent reoperation. The reason for reoperation was undercorrection in 78 cases (49.0\%), disappearance of the dimple in 62 cases (38.9\%), and overcorrection in nine cases $(5.6 \%)$. Five patients $(3.1 \%)$ had their stitches removed to eliminate the created dimple because they changed their minds, and five patients (3.1\%) had their stitches removed because of infection. No patients reported complications after reoperation, and no other complications, such as hyperpigmentation or foreign body reaction, were observed.

Conclusions Safe surgery with minimal complications and satisfying cosmetic results can be achieved via accurate knowledge of the relevant anatomy and its relationship with dimples, as well as appropriate surgical methodology.

Keywords Surgery, plastic / Face / Cheek / Surgical procedures, operative
Correspondence: Jeong Su Shim Department of Plastic and Reconstructive Surgery, Daegu Catholic University School of Medicine, 33 Duryugongwon-ro 17-gil, Nam-gu, Daegu 42472, Korea Tel: +82-53-650-4581

Fax: +82-53-650-4584 E-mail:21csue@hanmail.net

\section{INTRODUCTION}

A dimple is a small depression in the cheek or near the mouth that appears when smiling due to the contraction of the mimic muscles, particularly the zygomaticus major. Because dimples can enrich facial expressions, many people find them attractive.
As dimples are innate, dimple creation surgery is sometimes desirable to those who are born without them.

Essentially, dimple creation surgery involves creating a region of scar tissue through a suture between the buccinator muscle and the dermis. When the authors searched the existing literature on dimple surgery, an insufficient amount of research was 
found. The principles of dimple surgery do not vary greatly, but tips for an easy and effective surgical process are not frequently shared among physicians. Therefore, we intend to share some surgical tips for convenient surgery and consistent results, in addition to possible complications and surgical outcomes.

\section{METHODS}

We retrospectively analyzed the medical records of patients who underwent dimple creation surgery at our clinic. This study was approved by the Institutional Review Board of Daegu Catholic University Medical Center (CR-20-001). Informed consent was provided by all patients.

\section{Patient group}

This study included 2,048 patients who underwent dimple creation surgery at our cosmetic surgery clinic between April 2010 and June 2014. None of these patients displayed any scarring from wounds or tumor removal in the central face during the presurgical evaluation. Medical records were used to identify the age and sex of the patient, the location of dimple creation, any postsurgical complications, reoperation, and the reason for reoperation.

\section{Surgical method}

Patients were asked to smile in either a sitting or a standing position so that we could mark the point at which the new dimple would be created. As described in the Discussion section, the position was primarily determined based on the patient's desire in conjunction with the typical position of dimple creation. If the patient was unable to determine the point, the work of BooChai [1] was referenced to determine it. Betadine solution was diluted to wash the mouth, which was then rewashed with saline solution or distilled water. Before the procedure, betadine was also used to resterilize the marked area of the skin and the expected surgical site on the mucous membrane in the mouth. The authors used modified chalazion forceps (Medical Land, Seoul, Korea) to accurately match the marked area on the cheek with that on the buccal mucosa. The oval-shaped tip of the forceps was held with its center aligned with the mark on the skin, and the buccal mucosa was inverted to mark the incision area on the mucous membrane. A solution of $1 \%$ lidocaine with 1:200,000 epinephrine was mixed and injected into the skin and mucosa. While holding the forceps, we used a no. 11 blade to make a 2- to 3-mm incision on the buccal mucosa and opened it slightly with Metzenbaum scissors. The opening of the Stensen duct was easily visible to the naked eye. While making the incision, one may need to be cautious to avoid damaging the
Stensen duct; however, the position of most incisions is anterior and inferior from the duct opening. Based on the size of the desired dimple in relation to the marked position on the skin, two positions $0.3-0.8 \mathrm{~cm}$ apart were marked for insertion of the needle. A 4-0 nylon suture was begun at one marked area on the skin of the cheek and passed through the dermis at the other marked point. The needle was cut off from the suture. Then, the two ends of the remaining thread were connected separately from the straight needle, and the two ends of each thread were removed from the incision window of the oral mucosa and knotted in the mucous membrane (Fig. 1).

The depth of the dimple was controlled through the tension in

Fig. 1. Surgical method of dimple creation

(A) From the site marked on the skin side (although some variation can exist depending on the desired dimple size), at average intervals of 0.3 to $0.8 \mathrm{~cm}$, two sites were punctured vertically up to the dermis layer using 21-gauge needles. (B) Using a 4-0 nylon suture, from the punctured site in the skin side, a needle was passed through the dermis and inserted into the other puncture site. (C) Starting from the area where the needle emerged, the needle was passed through the incision site in the mucosa of the oral cavity. The two ends of the suture emerged from the incision site of the mucosa and were knotted.
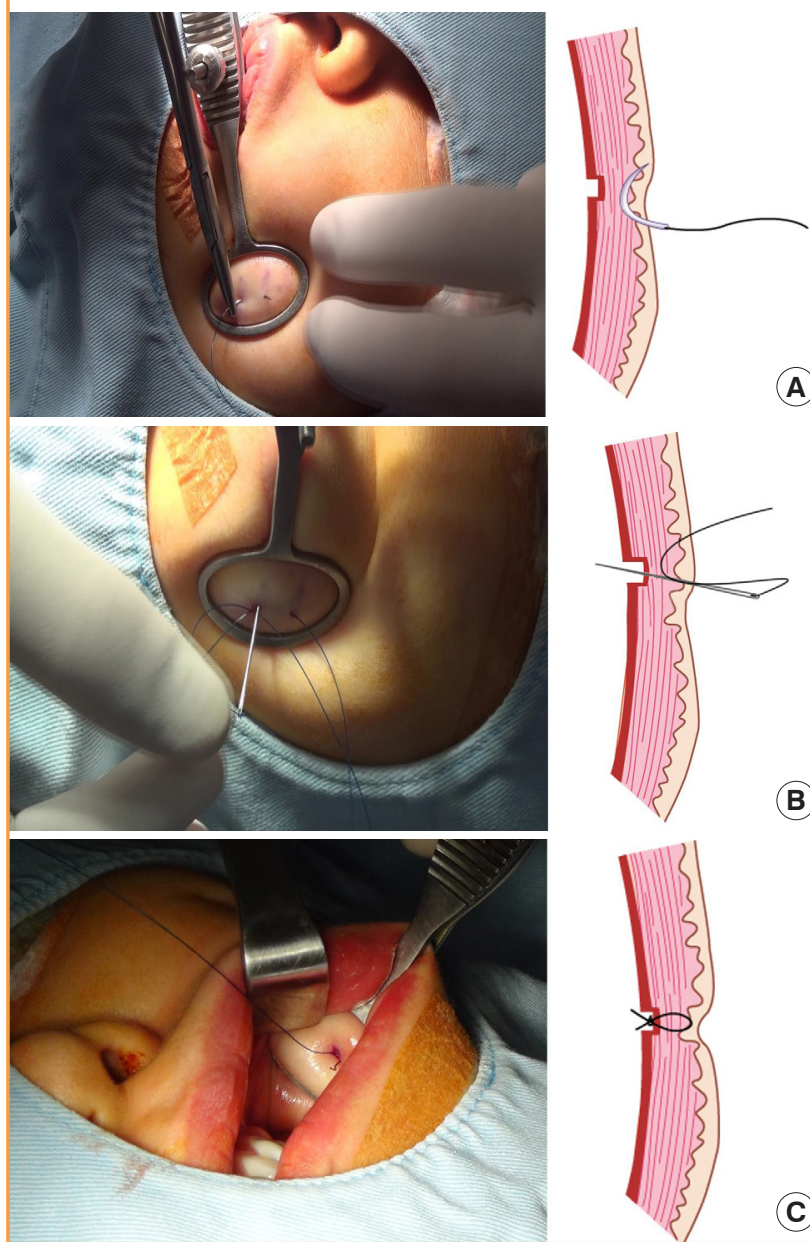

(B)

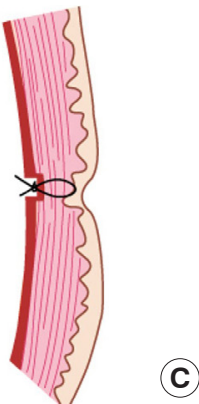


the knot. A stronger knot corresponds to a deeper dimple, and vice versa. Lastly, the incision site of the buccal mucosa was sutured with a chromic 5-0 suture (Supplemental Video 1).

For the creation of dimples in the corners of the mouth, the positioning and design primarily depended on the patient's desire. In most cases, a cross-stitch suture was made with a needle without an incision [2].

No particular dressing was used on the surgical site.

\section{RESULTS}

The study included a total of 2,048 subjects, the ages of whom ranged from 18 to 57 years (mean, 26 years). Of these, 83 patients $(4.05 \%)$ were $<20$ years old, 1,564 patients $(76.37 \%)$ were 20 to 29 years old, 316 patients (15.43\%) were 30 to 39 years old, and 85 patients ( $4.16 \%$ ) were $>40$ years old. Thus, the largest age group consisted of patients in their 20s. The patients included 26 men (1.2\%) and 2,022 women (98.85\%). A total of 655 patients $(31.9 \%)$ underwent surgery on both cheeks, while 539 (26.3\%) and 849 (41.4\%) patients underwent surgery on the right and left cheeks alone, respectively. Two patients $(0.09 \%)$ had dimples created on both mouth corners, while one patient $(0.04 \%)$ had a dimple created on the right mouth corner only. Two patients $(0.09 \%)$ underwent dimple creation surgery on the left mouth corner only (Table 1 ). The average follow-up period was 7.35 months (range, 1-78 months).

Potential initial complications include bleeding, hematoma, edema, infection, and nerve injury. Bleeding occurred at the time of incision in some cases, but was resolved in a few minutes through compression. Hematoma was not observed. Postsurgical edema was not considered a complication because it disappeared within 2 weeks in all cases. Eight patients visited the hospital for infection, which was treated with incision and drainage. All infections occurred within 2 weeks of surgery. Stitches were removed in five cases. No cases of nerve injury were observed. The scar on the puncture site on the skin faded to some extent by $8-12$ weeks from surgery, and natural-looking dimples

\section{Table 1. Location distribution of facial dimple surgery}

\begin{tabular}{|lc|}
\hline Location & No. of patients (\%) \\
\hline Both cheek & $655(31.98)$ \\
Right cheek & $539(26.32)$ \\
Left cheek & $849(41.46)$ \\
Both mouth corner & $2(0.09)$ \\
Right mouth corner & $1(0.04)$ \\
Left mouth corner & $2(0.09)$ \\
Total & $2,048(100)$ \\
\hline
\end{tabular}

formed by 20-24 weeks.

Potential follow-up complications include overcorrection, undercorrection, disappearance, and asymmetry, which are largely cosmetic issues. Reoperations due to these complications were investigated.

Of the 2,048 patients, 159 (7.7\%) underwent reoperation for the following reasons: undercorrection (78 cases, $49.0 \%)$, disappearance of the dimple (62 cases, $38.9 \%$ ), overcorrection (nine cases, 5.6\%), infection that required removal of stitches (five cases, 3.1\%), and removal of stitches to eliminate the dimple upon a change of mind (five cases, 3.1\%) (Table 2). Of the cases involving stitch removal due to a change of mind, three cases were completed in the first month after dimple creation surgery, while in two cases, the stitches were removed after several months. Asymmetry may occur in cases of bilateral dimple creation. In this study, no differences in dimple positioning were observed, but some cases did involve undercorrection or overcorrection on one side. Therefore, such cases were included in the above counts of reasons for reoperation.

No patients reported complications after reoperation, and no

\section{Table 2. The reason of reoperation}

\begin{tabular}{|lc|}
\hline Reason of reoperation & No. of patients (\%) \\
\hline Undercorrection & $78(49.0)$ \\
Disappear & $62(38.9)$ \\
Overcorrection & $9(5.6)$ \\
Infection of stitch & $5(3.1)$ \\
Simple change in mind & $5(3.1)$ \\
Totala) & $159(100)$ \\
\hline a) Reoperation of facial dimple surgery $=7.7 \%(159 / 2,048)$. \\
\hline
\end{tabular}

\section{Fig. 2. Case 1}

A 26-year-old woman is shown before and 4 months after dimple creation on the left side. $(A, B)$ Preoperative view. $(C, D)$ Postoperative view at 4 months after surgery.
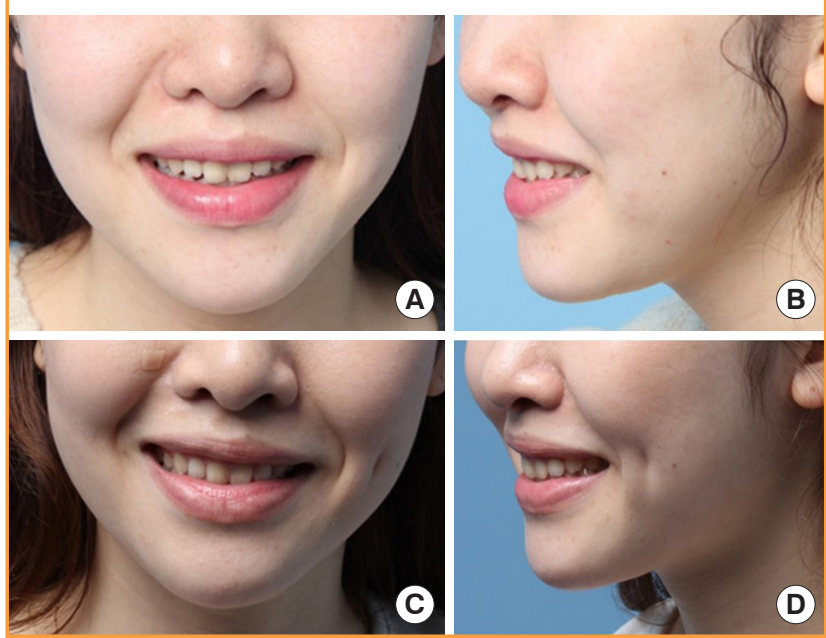


\section{Fig. 3. Case 2}

A 28-year-old woman is shown before and 12 months after bilateral dimple creation. (A, B) Preoperative view. (C, D) Postoperative view at 12 months after surgery.
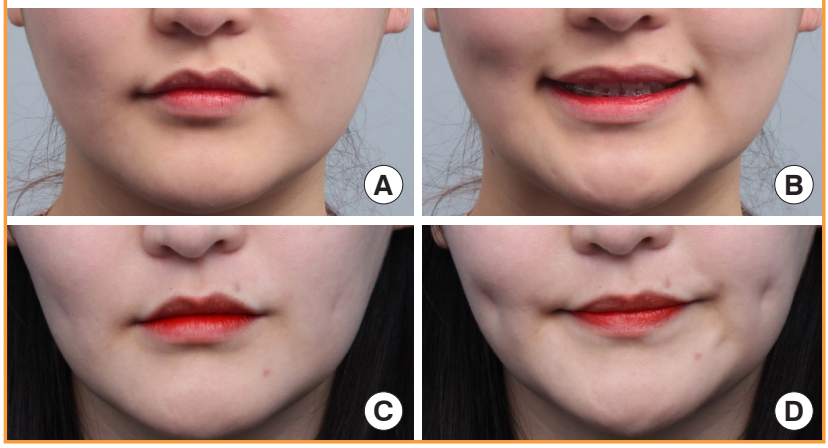

complications, such as hyperpigmentation or foreign body reaction, were observed during the follow-up period (Figs. 2-4). The depth of the dimple varies depending on the facial expression; for instance, as shown in Fig. 4, the dimple appears deeper when smiling with the mouth closed and the corners of the mouth tightened than when smiling widely and showing the teeth.

\section{DISCUSSION}

Dimples that are visible when smiling are inherited as an autosomal dominant trait, which is present on chromosome 16 for cheek dimples and chromosome 5 for chin dimples. Dimple formation may be affected by environmental and other factors [3] .

Dimples are formed by the bifid zygomaticus major, which is a variation of the zygomaticus major muscle [4,5]. Meta-analytical studies in anatomy have reported that the origin of the bifid zygomaticus major muscle is mainly in the zygomatic bone and its surroundings, and the insertion is divided into the superior bundle and inferior bundle. The described attachment region varies slightly by study. The prevalence of the bifid zygomaticus major has been found to be $22.7 \%$ in the general population, with a $34 \%$ prevalence among Americans, $27.4 \%$ among Asians, and $12.3 \%$ among Europeans [6]. Other studies have reported a possible impact of the location and form of the lateral band of the orbicularis oculi muscle on dimple formation [7]. The effect of the risorius muscle on the position of cheek dimples may also be considered, but no anatomical research has been performed on this topic.

Dimples are perceived to be negative by some cultures; however, they are thought to be attractive in South Korea and other countries, and many people desire to have them. The demand for dimple surgery is high among Asians [8]. Regarding the site of dimple creation, our surgical results showed that patients pre-

\section{Fig. 4. Case 3}

(A) A 34-year-old woman is shown 78 months after bilateral dimple creation. (B) Faint marks are present on both cheeks, but they are difficult to see. (C) A close-mouthed smiling expression. The dimple appears slightly deeper than when the patient is smiling while showing teeth. (D) A wide smile. The dimple looks less deep when smiling widely while showing teeth than when smiling with the mouth closed.
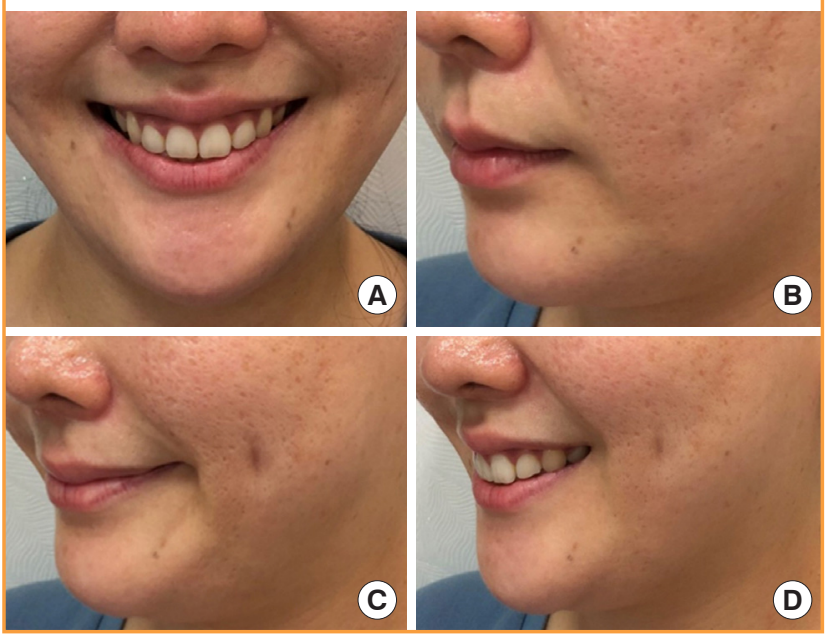

ferred the cheek over the chin and one side over both sides. They also preferred the left side over the right side.

Dimples may give different impressions depending on their location. The desired position of dimples is subjective, and as a result, opinions vary on their ideal position. The most commonly used reference for the location of cheek dimples for surgery is that of Boo-Chai [1]. He stated that the best location, from a cosmetic viewpoint, is the bisection of a perpendicular line extended downward from the lateral canthus and a horizontal line drawn from the angle of the mouth when the patient smiles. This information guided us in marking the point for dimple creation while the patient is smiling, and it is largely consistent with the position observed when the face is returned to a normal expression. Other opinions exist; Argamaso [8] argued for an ideal dimple position 3-3.5 cm from the oral commissure on the line connecting the oral commissure and earlobe. Similarly for cheek dimples, some surgeons have described using a position on the intersecting point that is more forward than the one mentioned by Boo-Chai [1] by lowering the vertical line based on the lateral limbus and not the lateral canthal angle. In one relevant study, new guidelines were suggested for dimple positioning by comparing cases involving different surgical positions from that described by Boo-Chai [9]. We prefer the reference by Boo-Chai because these dimples are mostly located $2.5-3 \mathrm{~cm}$ away from the oral commissure. However, because we did not focus on this topic in this study, further studies are needed to obtain accurate findings regarding ideal dimple posi- 
tioning.

Surgical procedures for dimple creation have been previously described by several authors. In most cases, dimple creation involves creating a region of scar tissue via sutures between the buccinator muscle and the dermis [10]. Many techniques have already been introduced, including making a cross-stitch suture with a needle without incision, using small incisions, creating a defect in the oral mucosa, using bolster sutures, and others.

The classic method as described by Boo-Chai [1] involves administering local anesthesia and making a short incision of approximately $3 \mathrm{~mm}$ in the oral mucosa at the expected position of the dimple. Through this incision, a needle with a 4-0 nylon suture is taken out of the skin surface and then reinserted into the same hole. The needle is passed through $5 \mathrm{~mm}$ of dermis and is then removed from the skin. Then, the needle is reinserted into the same hole and removed from the incision in the oral mucosa, which is then sutured.

The method described by Argamaso [8] involves piercing the needle through the dimple creation area on the skin after local anesthesia and using an 8-mm biopsy punch to cut the tissue to below the skin from the membrane area pierced by the needle. The mucosa and skin are then sutured through this wound to create the dimple.

While similar to the classic method by Khoo, our method is modified slightly to obtain more consistent and predictable results. We used modified chalazion forceps (Medical Land) that are designed for dimple creation. This instrument is also called a dimple forceps and adjusts the chalazion open-ring forceps to allow for better holding of the oral mucosa and cheek skin. This instrument enabled us to find the dimple position marked on the skin and the buccal mucosa to increase the surgical accuracy. Unlike cheek dimples, the dimples in the mouth corners were created mostly by making a cross-stitch suture using a needle without an incision. This technique was preferable because mouth corner dimples are smaller than cheek dimples.

In general, the advantage of our procedure is that it is easier to control the location, depth, and size of the dimples than to make an incision only within the intraoral mucosa. The disadvantage is that a scar is visible for a period of time after surgery. However, this scar fades to become invisible over time.

The depth of the dimple is controlled by the strength of the knot during surgery. However, the use of knotting does not sufficiently control the depth. If the patient wishes to have a deep dimple, the incision in the oral mucosa is made slightly anterior of the thread-fixing point on the skin to deepen the dimple. This is thought to relate to the vector of movement of facial muscles and soft tissue when the patient smiles. When smiling, the mouth corner and cheek move in the posterior superior direc- tion, and making the incision site in the opposite direction can help deepen the dimple. Depth control of the dimples particularly impacts the symmetry of bilateral dimple surgery. In this regard, a further consideration is that the depth of the dimples may vary depending on the facial expression, as shown in Fig. 4. When we close our mouths and smile, the risorius muscle usually pulls the corners of the mouth outwards, and when we laugh and show our teeth, the zygomaticus major and minor muscle usually work to raise the corners of the mouth up. As shown in Fig. 4, the dimple was made in the risorius muscle area, yielding the described result. In order to make the dimple look deeper when laughing robustly, it is necessary to verify the direction of action of each facial muscle before surgery.

Dimple asymmetry after surgery may be caused by a difference in the depth or length of the dimples rather than by a difference in position, and most often, asymmetrical-seeming dimples are caused by a difference in depth. This is thought to be caused by differences in adhesion on both sides after surgery and the degree of habitual use of the facial muscles used when the patient smiles. When creating dimples on both sides, it is important to first design the preoperative position symmetrically. During surgery, the muscles of the both sides are sufficient to tie up the knot and do not differ greatly in strength between the sides. Adjustment of the dimple depth is more closely related to the direction of the thread than the force of the tie. If the asymmetry is severe, reoperation may be needed. The degree of symmetry (the degree of depth similarity) of the dimples can also be complemented by reoperation.

As discussed above, the opening of the Stensen duct (which is visible to the naked eye) can be visually confirmed by holding the oral mucosa and skin with the forceps. The Stensen duct opening can be observed superior and posterior to the incision. In this fashion, damage to this structure can be avoided in advance. Jones et al. [11] anatomically demonstrated the safety zone needed to avoid damage to the Stensen duct in their anatomical discussion.

Complications were rare in our patients. According to our results, in practice, postsurgical complications are rare in the initial stage, making postsurgical satisfaction a more relevant consideration in dimple creation surgery than complications.

Some examples of initial complications after dimple creation surgery include hyperpigmentation, foreign body reaction, and hemorrhage, which are rare. Depending on the anatomical location, damage may also occur to the buccal nerve, a facial nerve branch. Damage to this nerve may paralyze the buccinators and cause abnormal motion of the nose and upper lip while smiling. However, most dimples are positioned at the terminal branch of the buccal nerve, which has a number of branches, thus making 
it difficult for clinical symptoms of such damage to be observed. Though rare, infection is a possible complication. If initial treatment is incorrect in case of infection, a scar may remain in an undesirable shape. If infection is suspected, the treating clinician should consider the possibility of not only bacterial infection but also fungal infection [12].

Late complications generally do not result in an exterior scar. In most cases, dimples fade, weaken, or are formed more strongly than desired. Regarding asymmetry, we found no cases of asymmetric location, meaning that cases of asymmetry generally result from a difference in the depths of the dimples. Before bilateral dimple surgery, patients should be fully informed of the possibility of asymmetry.

Dimple creation surgery is frequently and consistently demanded as a simple procedure for those who crave a more attractive smile. Nevertheless, relatively few studies exist regarding follow-up monitoring, complications, and corrective surgery. Fundamentally, accurate knowledge of the surgical methodology and the relationship between the relevant anatomy and dimples is required to minimize surgical complications and obtain satisfactory cosmetic results. We hope to provide assistance to beginners by sharing our surgical methods and results with other plastic surgeons.

\section{NOTES}

\section{Conflict of interest}

No potential conflict of interest relevant to this article was reported.

\section{Ethical approval}

The study was approved by the Institutional Review Board of Daegu Catholic University Medical Center (IRB No. CR-20001) and performed in accordance with the principles of the Declaration of Helsinki. Written informed consents were obtained.

\section{Patient consent}

The patients provided written informed consent for the publication and the use of their images.

\section{Author contribution}

Conceptualization: JM Jung, JH Park, JS Shim. Data curation: JS Shim. Formal analysis: JS Shim. Methodology: JM Jung. Project administration: JM Jung. Visualization: JM Jung, JH Park. Writing - original draft: JH Park. Writing - review \& editing: JH Park.

\section{ORCID}

Jae Min Chung

Joo Hyuk Park

https://orcid.org/0000-0002-8893-5876

Jeong Su Shim

https://orcid.org/0000-0002-2482-8131

https://orcid.org/0000-0002-8458-4415

\section{Supplementary material}

Supplemental Video 1. Surgical method of dimple creation surgery. Supplemental data can be found at: https://doi.org/10.5999/ aps.2020.00661.v001.

\section{REFERENCES}

1. Boo-Chai K. The facial dimple: clinical study and operative technique. Plast Reconstr Surg Transplant Bull 1962;30: 281-8.

2. Bao S, Zhou C, Li S, et al. A new simple technique for making facial dimples. Aesthetic Plast Surg 2007;31:380-3.

3. Omotoso GO, Adeniyi PA, Medubi LJ. Prevalence of facial dimples amongst South-western Nigerians: a case study of Ilorin, Kwara State of Nigeria. Int J Biomed Health Sci 2010; 6:241-4.

4. Pessa JE, Zadoo VP, Garza PA, et al. Double or bifid zygomaticus major muscle: anatomy, incidence, and clinical correlation. Clin Anat 1998; 11:310-3.

5. Pessa JE, Zadoo VP, Adrian EK Jr, et al. Variability of the midfacial muscles: analysis of 50 hemifacial cadaver dissections. Plast Reconstr Surg 1998;102:1888-93.

6. Phan K, Onggo J. Prevalence of bifid zygomaticus major muscle. J Craniofac Surg 2019;30:758-60.

7. Park JT, Youn KH, Hur MS, et al. Malaris muscle, the lateral muscular band of orbicularis oculi muscle. J Craniofac Surg 2011;22:659-62.

8. Argamaso RV. Facial dimple: its formation by a simple technique. Plast Reconstr Surg 1971;48:40-3.

9. Almaary HF, Karthik R, Scott C. New landmarks for the surgical creation of dimples based on facial form. J Clin Aesthet Dermatol 2018;11:22-6.

10. Thomas M, Menon H, D’Silva J. Improved surgical access for facial dimple creation. Aesthet Surg J 2010;30:798-801.

11. Jones NI, Gamboa G, Bhatt K. Transoral buccinator-pexy (TBP). Ann Plast Surg 2014;72:S132-4.

12. AbdullGaffar B, Ghandoor K, Ahli Q. Actinomycotic sinus of the buccal mucosa: a rare complication of cheek dimple creation. Aesthet Surg J 2014;34:NP80-2. 